\title{
Determining Conjugate Points of An Aerial Photograph Stereopairs Using Separate Channel Mean Value Technique
}

\author{
Andri Hernandi ${ }^{1}$, D. Muhally Hakim ${ }^{1}$, Irawan Seomarto ${ }^{1}$, Agung Budiharto ${ }^{1}$ \& \\ Emalia $^{2}$ \\ ${ }^{1}$ Faculty of Earth Sciences and Technology- ITB \\ ${ }^{2}$ Alumnus of Faculty of Earth Sciences and Technology- ITB
}

\begin{abstract}
In the development of digital photogrammetric system, automatic image matching process play an important role. The automatic image matching is used in finding the conjugate points of an aerial photograph stereopair automatically. This matching technique gives quite significant contribution especially in the development of 3D photogrammetry in an attempt to get the exact and precise topographic information during the stereo restitution. There are two image matching methods that have been so far developed, i.e. the area based system for gray level environment and the feature based system for natural feature environment. This research is trying to implement the area based matching with normalized cross correlation technique to get the correlation coefficient between the spectral value of the left image and its pair on the right. Based on the previous researches, the use of color image could increase the quality of matching. One of the color image matching technique is known as Separate Channel Mean Value. In order to be able to see the performance of the technique, a number of sampling areas with various different characteristics have been chosen, i.e. the heterogeneous, homogeneous, texture, shadow, and contrast.
\end{abstract}

The result shows the highest similarity measure is obtained on heterogeneous sample area at size of all reference and search image, i.e. (11 pixels x 11 pixels) and ( 23 pixels $\times 23$ pixels). In these area the correlation coefficient reached more than 0.7 and the highest percentage of similarity measure is obtained. The average of total similarity measure of conjugate images in the sampling image area only reach about $41.43 \%$ of success. Therefore, this technique has a weakness and some treatment to overcome the problems is still needed.

Keywords: area-based matching; conjugate points; correlation coefficient; image matching; photogrammetry.

\section{$1 \quad$ Introduction}

A fundamental problem in photogrammetric system is the reconstructing of the 3D data from an aerial photography stereopairs. For example, part of photogrammetric process such as relative orientation, absolut orientation, aerial 
triangulation, orthophoto and digital terrain model (DTM) generation. Unfortunately, the analogue approach of those process are time consuming and the results are depending upon the operator skill. To overcome the problems the human tasks are gradually replaced by the digital computer. This paper described part of the digital system to establish an automatic photogrammetric process, i.e. the utilization of digital image processing.

Human vision has the capability to search conjugate points or objects which appear in overlaping area of an aerial photograph stereopair e.g. determination of six pairs of conjugate otto von gruber points in left and right photograph in relative orientation process. To that end, is actually a searching process base on pattern recognition method. The process of finding out the conjugate points (or objects) in two or more overlapping photographs is a fundamental process in photogrammetric system. In digital domain, this process can be done automatically, known as image matching method [1].

The developments of image matching technique in the field of photogrammetry has quite long history. First experiments started in the fifties by Hobrough [2] until reseachers have been trying to make every single effort to find out the best image matching technique. Despite considerable effort, no general solution was found. A question arises among the researchers how the human vision is able to find conjugate points easily without a lot of effort. This is just an example in showing the complexity of the human vision in solving the problem easily.

The image processing domain particularly in photogrametric application related to the image matching can be divided into two : the area-based and the feature based matching methods. Area-based matching method is more popular compare to feature-based matching method, because of historical reasons. Area-based matching is associated with matching the gray values. The gray level distribution of small areas of two images, called image patches, is compared by correlation or least-square techniques.

Since the images contain of full 24-bit color information, the above techniques could be modified when applying the gray-value image. Two ways have been inserted into the correlation techniques, resulting in one single similarity value. The other approach calculates each channel separately and allows individual assessment. This research applying a mean value of three channels as similarity value. This technique has been known as Separate Channel Mean Value Correlation [3]. Kuzu [3] has implemented this technique for volumetric object reconstruction purpose. This technique has been used to refine the visual of the object reconstruction. Furthermore, this technique has been experimented in finding conjugate points or natural objects in a number of sample of aerial photographs having different characteristics. 
The main objective of this research is investigating method to implement image matching procedure, particulary color information consideration in an images, using separate channel mean value correlation techniques on aerial photograph. The result of this research are expected to give real contribution for improvement image matching methods which can be used for wide applications of automatic geographic extraction in digital photogrametric (DTM, topographic features compilation, etc.). Particularly for supporting the early process of photogrametric method those are determination six otto von gruber points.

\section{$2 \quad$ Area-Based Matching Method}

Area-based matching usually work based on local windows or image patches. The image patches consist of reference image, search image and sub-search image. The reference image is usually kept in a fixed position within one of the selected image. The sub-search image is a moving image patches in the search image having equal size with reference image. The sub-search images are compared to the reference image. The comparison is performed with different similarity measure based on correlation or least-squares techniques [4]. The concept of area-based matching showed in Figure 1.

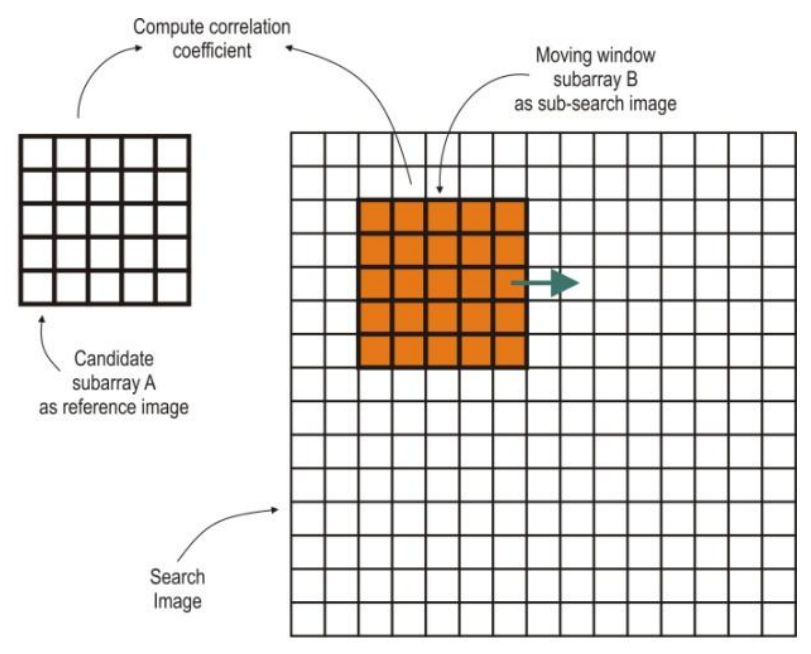

Figure 1 Concept of area-based matching.

\subsection{The Correlation Technique}

The correlation is the most familiar technique in finding the conjugate points in photogrammetric application. The main idea of the correlation technique is to measure the similarity between reference image and search image by computing 
the correlation coefficient. The correlation coefficient $(\rho)$ is computed using the following formula:

$$
\rho=\frac{\sigma_{L R}}{\sigma_{L} \cdot \sigma_{R}}
$$

where :

$\rho \quad:$ correlation coefficient $(-1 \leq \rho \leq+1)$.

$\sigma_{L R} \quad$ : covariance of image patches $\mathrm{L}$ (reference image) and $\mathrm{R}$ (subsearch image)

$\sigma_{L} \quad:$ standard deviation of image patch L (reference image)

$\sigma_{R} \quad$ : standard deviation of image patch $\mathrm{R}$ (sub-search image)

Introducing the image function $g_{L}\left(x_{i}, y_{j}\right), g_{R}\left(x_{i}, y_{j}\right)$ for the left and right image pacthes (reference and sub-search image) and their means $\bar{g}_{L}\left(x_{i}, y_{j}\right), \bar{g}_{R}\left(x_{i}, y_{j}\right)$ to equation (1) produced :

$$
\rho=\frac{\sum_{i=1}^{n} \sum_{j=1}^{m}\left(\left(g_{L}\left(x_{i}, y_{j}\right)-\bar{g}_{L}\left(x_{i}, y_{j}\right)\right) \times\left(g_{R}\left(x_{i}, y_{j}\right)-\bar{g}_{R}\left(x_{i}, y_{j}\right)\right)\right)}{\sqrt{\sum_{i=1}^{n} \sum_{j=1}^{m}\left(g_{L}\left(x_{i}, y_{j}\right)-\bar{g}_{L}\left(x_{i}, y_{j}\right)\right) \times \sum_{i=1}^{n} \sum_{j=1}^{m}\left(g_{R}\left(x_{i}, y_{j}\right)-\bar{g}_{R}\left(x_{i}, y_{j}\right)\right)}}
$$

The correlation coefficient $(\rho)$ is determined for every row and colum position of the sub-search image within the search image. The next step is to determine the position of the sub-search image having the maximum correlation coefficient.

\subsection{Separate Channel Mean Value}

Since the image contain color information, the equation (2) can be modified using a mean value of three channels as single similarity measure. This technique is knowns as Separate Channel Mean Value Correlation [3].

Let $\rho^{\text {red }}, \rho^{\text {green }}$ and $\rho^{\text {blue }}$ are the correlation coefficients for red, green and blue respectively; furthermore :

$$
\rho^{\text {Total }}=\frac{\rho^{\text {red }}+\rho^{\text {green }}+\rho^{\text {blue }}}{3}
$$

$\rho^{\text {Total }}$ is the total corelation coefficient as one single similarity measure calculated from mean value of three channels. Having computed for all position of the sub-search image then the total correlation coefficient is chosen from the 
one having the maximum correlation coefficient. Two objects on the image can be regarded as two same objects if the correlation coefficient is between 0.7 to $1.0[5]$.

\section{Experimental Works}

For the purpose of this research two overlapped aerial color photographs of Sabuga area - ITB campus has been used. The overlapping area is considered having different terrain charactertistics, i.e. homogeneous, heterogenous, shadow, texture density, and contrast. This aerial photographs have been taken from aerial photography mission by Cessna aircraft with 68\% overlap. See Figure 2.

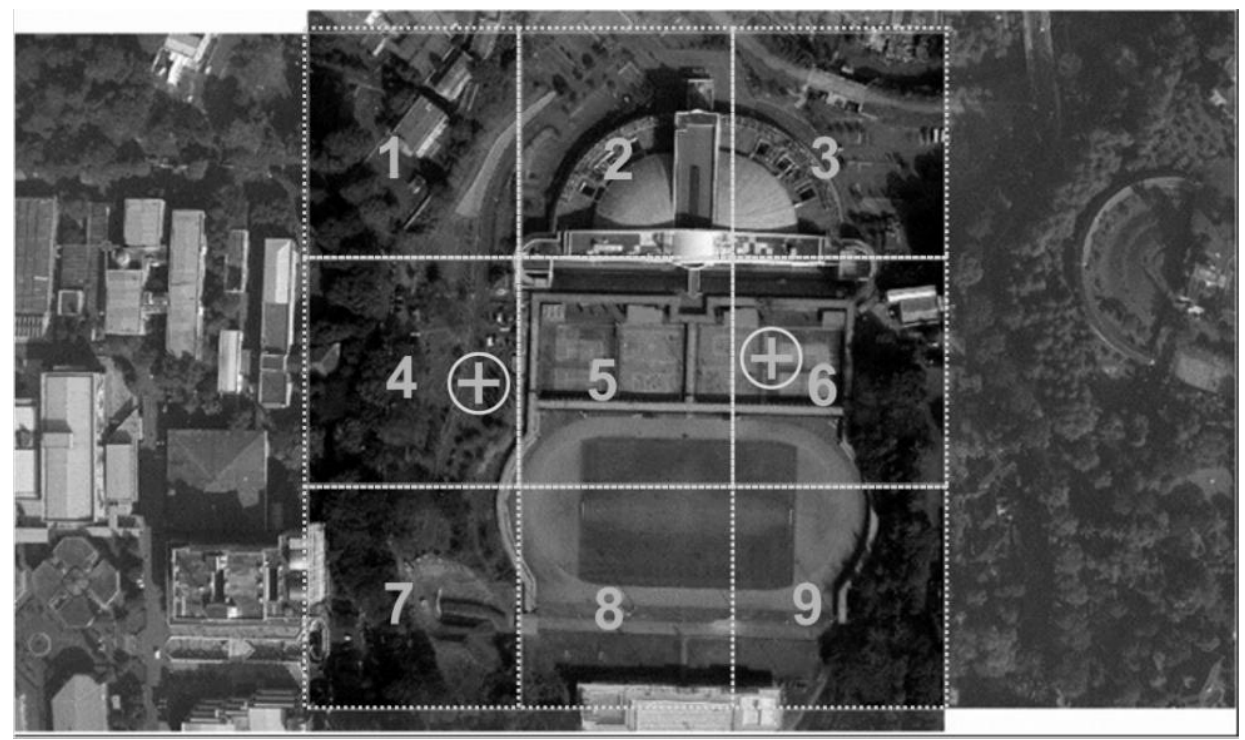

Figure 2 Research area on overlaped aerial photograph of Sabuga.

The sistematic steps of work in carrying out this experiment are as follow:

1. Identifying approximate location of conjugate points based on various different characteristic (homogeneous, heterogenous, shadow, texture density, and contrast) and distance from principle points on an aerial photograph stereopairs.

2. Exctracting image patches (reference image and search image) with different sizes from aerial photograph stereopairs.

3. Computing correlation coefficient of each image patches using separate channel mean value correlation technique. 
4. Analyze the similarity measure using maximum correlation coefficient of each image patches.

Determination of approximate location of the conjugate points on the overlapping photographs was done visually. The location of the points are chosen by considering the different object characteristics and having various distances from aerial photograph principal point. The overlapping area is devided into nine samples area, see Figure 2. For each pair of sample contains of three different size (11 pixels $\times 11$ pixels), ( 21 pixels $\times 21$ pixels), (33 pixels $x 33$ pixels) as reference image assosiated with three different size ( 23 pixels $x$ 23 pixels), (53 pixels $\times 53$ pixels), and ( 83 pixels $\times 83$ pixels) of search image respectively. In this experiment, the correlation coefficients are computed using Equation 3 for all grid samples, and the result can be seen Table 1.

Table 1 Maximum correlation coefficients and similarity measures to various different object characteristic.

\begin{tabular}{|c|c|c|c|c|c|}
\hline No. & $\begin{array}{c}\text { Object } \\
\text { Characteristic }\end{array}$ & $\begin{array}{c}\text { Size of } \\
\text { Reference } \\
\text { Image (pixels) }\end{array}$ & $\begin{array}{c}\text { Size of } \\
\text { Search } \\
\text { Image (pixels) }\end{array}$ & $\begin{array}{l}\text { Correlation } \\
\text { Coefficient }\end{array}$ & $\begin{array}{c}\text { Similarity } \\
\text { Measure } \\
(\%)\end{array}$ \\
\hline \multirow{3}{*}{1} & \multirow{3}{*}{ Homogenous } & $11 \times 11$ & $23 \times 23$ & 0.3993 & $0.00 \%$ \\
\hline & & $21 \times 21$ & $53 \times 53$ & 0.5211 & $12.50 \%$ \\
\hline & & $33 \times 33$ & $83 \times 83$ & 0.5532 & $25.00 \%$ \\
\hline \multirow{3}{*}{2} & \multirow{3}{*}{ Heterogenous } & $11 \times 11$ & $23 \times 23$ & 0.8486 & $87.50 \%$ \\
\hline & & $21 \times 21$ & $53 \times 53$ & 0.8044 & $87.50 \%$ \\
\hline & & $33 \times 33$ & $83 \times 83$ & 0.7778 & $75.00 \%$ \\
\hline \multirow{3}{*}{3} & \multirow{3}{*}{ Texture } & $11 \times 11$ & $23 \times 23$ & 0.485 & $17.65 \%$ \\
\hline & & $21 \times 21$ & $53 \times 53$ & 0.591 & $35.29 \%$ \\
\hline & & $33 \times 33$ & $83 \times 83$ & 0.6317 & $52.94 \%$ \\
\hline \multirow{3}{*}{4} & \multirow{3}{*}{ Shadow } & $11 \times 11$ & $23 \times 23$ & 0.3913 & $0.00 \%$ \\
\hline & & $21 \times 21$ & $53 \times 53$ & 0.4645 & $12.5 \%$ \\
\hline & & $33 \times 33$ & $83 \times 83$ & 0.6024 & $25.00 \%$ \\
\hline \multirow{4}{*}{5} & \multirow{4}{*}{\multicolumn{4}{|c|}{\begin{tabular}{ccc}
$11 \times 11$ & $23 \times 23$ & 0.6809 \\
$21 \times 21$ & $53 \times 53$ & 0.7441 \\
$33 \times 33$ & $83 \times 83$ & 0.7362 \\
\multicolumn{3}{r}{ Average of Total Similarity Measure }
\end{tabular}}} & $55.56 \%$ \\
\hline & & & & & $66.67 \%$ \\
\hline & & & & & $66.67 \%$ \\
\hline & & & & & $41.43 \%$ \\
\hline
\end{tabular}

As illustrated in Table 1, the average of total similarity measure of various different object characteristics is about $41,43 \%$ of success. The highest similarity measure is obtained on heterogenous area lead to about $75 \%-87.50$ $\%$, and the other characteristics area is conversely. In this heterogenitic area, the objects are easier to identify. 
Using separate channel mean value technique to be implemented to both various different characteristic shows average of total similarity measure of conjugate image reaches to about $41.43 \%$ of success.

\section{Conclusion}

Determining conjugate points of an aerial photograph stereopairs using separate channel mean value technique will be succeed if the method is applied to heterogenous area. The similarity measure obtained on heterogenous area is about $75 \%-87.50 \%$. These areas have maximum correlation coefficient of more than 0.7 and the highest procentage of similarity measure.

All experimental result using separate channel mean value technique show that the average of total similarity measure is only reach $41.43 \%$ of success. This mean that the technique may be has a weakness. One of the suggestions to get a better result is by aligning the two photographs with respect to the principal points such as epipolar line creation before matching process. Furthermore, the aerial photo images have also many weaknesses radiometrically (noise, misalignment, etc.), therefore radiometric corrections are needed.

\section{Acknowledgment}

These authors would like to thank to ITB Research Grant (No. 081j/K01.18/PL/2008) for financial support on this research.

\section{References}

[1] Schenk, T., Digital Photogrammetry, I, Terrascience, Laurelville OH, USA, 428 p, 1999.

[2] Hobrough, G., Automatic Stereoplotting, Photogrammetric Engineering and Remote Sensing, 25(5), pp. 763-769, 1959.

[3] Kuzu, Y., Photorealistic Object Reconstruction Using Color Image Matching, International Archives of Photogrammetry, Remote Sensing and Spatial Information Sciences, ISPRS Commision V Symposium, Corfu , 34(5), pp. 169-174, 2002.

[4] Ackermann, F., Digital Image Correlation: Perfomance and Potential Application in Photogrammetry, The Photogrammetric Record, 11(64), pp. 429-439, 1984.

[5] Wolf, P. R. \& Dewitt, B. A., Elements of Photogrammetry with Application in GIS ,3th Edition, McGraw-Hill, Boston, USA, $624 \mathrm{p}$, 2000. 